J. Clin. Chem. Clin. Biochem.

Vol. 18, 1980, pp. 591-593

\title{
Direktbestimmung von Aluminium in Serumproben mittels Inductively Coupled Plasma (ICP). Emissionsspektralanalyse
}

\author{
Von P. Schramel, A. Wolf
}

Gesellschaft für Strahlen- und Umweltforschung mbH, Physikalișch-Technische Abteilung, Neuherberg und

\section{B.J. Klose}

\section{Frauenklinik, Kreiskrankenhaus Erding}

(Eingegangen am 5. Dezember 1979/23. April 1980)

Zusammenfassung: Die Anwendbarkeit der ICP (Inductively Coupled Plasma)-Emissionsspektralanalyse für die direkte Bestimmung von Aluminium in Blutserum wird untersucht und die verwendete Methode beschrieben. Auf Grund der hohen Anregungstemperatur in einem Plasma treten dabei die von der Atomabsorptionsspektroskopie her bekannten chemischen Matrixeinflüsse nicht auf, so daß ein sehr empfindlicher und gut reproduzierbarer Nachweis möglich ist.

\section{Determination of aluminium in blood-serum by Inductively Coupled Plasma (ICP)-Spectroscopy}

Summary: The application of ICP emission spectroscopy for the direct determination of aluminium in blood serum was investigated and the method with all important parameters is described. The well known matrix interferences in atomic absorption spectroscopy do not exist in ICP spectroscopy, due to the very high excitation temperature of the sample of about $8000 \mathrm{~K}$. It is therefore possible to perform very sensitive and reproducible measurements.

\section{Einleitung}

Die Bestimmung von Aluminium in menschlichem und tierischem Blutserum gewinnt immer mehr an Bedeutung, da bisher über die Aufnahme, Verteilung und Wirkungsmechanismen dieses Elements im Organismus nur sehr wenige und unvollständige Daten vorliegen. Andererseits gelangt aber $\mathrm{Al}$ auf vielfältige Weise in den menschlichen Organismus. Als Beispiele seien hier angeführt:

1. Al wird in großen Mengen bei der Herstellung und bei der Verpackung von Nahrungsmitteln verwendet,

2. Al-Sulfat wirid teilweise in der Aufbereitung von Trinkwasser zur Sedimentierung eingesetzt, und

3. Al und desssen Verbindungen werden als Therapeutika, z. B. zur Behandlung der Hyperphosphatämie bei Nierenerkrankungen und zum Senken der Magensäurekonzentration, z. B. bei Gastritis, verwendet.

Vor allem der Zusatz von Al zur Dialyseflüssigkeit, um Phosphate an $\mathrm{Al} \mathrm{zu}$ binden und aus dem Körper auszuscheiden, wobei die Resorption des Aluminium und dessen Wirkung bzw. Wechselwirkung in den verschiedenen Organen nicht geklärt ist $(1,2,3)$, machen eine Uberwachung bzw. eine eingehende Untersuchung der Al-Konzentration im Blutserum notwendig. Es gibt sogar Hinweise dafür, daß das ,Dialyse-Enzephalopathie-Syndrom" durch eine Al-Intoxikation hervorgerufen wird (4).

Alle diese Fragestellungen machen eine empfindliche und genaue Methode zur Bestimmung von $\mathrm{Al}$ in Gewebe und Körperflüssigkeiten notwendig. Bisher wurden für die Bestimmung der Al-Konzentration im Blutserum hauptsächlich zwei Verfahren eingesetzt:

1. die flammenlose Atomabsorptionsspektroskopie und

2. die instrumentelle Neutronenaktivierungsanalyse.

Die instrumentelle Neutronenaktivierungsanalyse setzt das Vorhandensein eines geeigneten Reaktors $\left(\mathrm{D}_{2} \mathrm{O}\right.$ moderiert wegen Störreaktionen durch schnelle Neutronen) sowie eines geeigneten Meßplatzes zur GammaSpektroskopie in unmittelbarer Reaktornähe (wegen 
der kurzen Halbwertszeit von ${ }^{28} \mathrm{Al}$ von $2,3 \mathrm{~min}$ ) voraus und ist deshalb in den meisten Fällen für die Routine nicht einsetzbar.

Der Nachweis mit der flammenlosen Atomabsorptionsspektroskopie ist $z$ war möglich und wird in der Routine auch meist angewendet, doch treten oft erhebliche Schwierigkeiten bei der Pipettierung von Serum in das Graphitrohr auf, so daß die erhal tenen Ergebnisse meist keine befriedigende Reproduzierbarkeit aufweisen. Auch treten bei diesem Verfahren aufgrund der notwendigen hohen Temperatur im ,thermischen Vorbehandlungsschritt" im Graphitrohr bereits vor der Atomisierung Verluste an Aluminium auf, jedoch nur bei Anwesenheit von Chlor und den sich dadurch bildenden flüchtigen Chlorverbindungen (5). Aus diesen Gründen wurde versucht, die ICP-Emissionsspektralanalyse für diese Untersuchungen einzusetzen.

\section{Material und Methoden}

\section{Proben}

Zur Erarbeitung und zum Testen dieser Methode standen 20 verschiedene Serumproben eines Normalkollektivs einer anderen Untersuchungsreihe zur Verfügung. Die Proben wurden tiefgefroren $\left(-25^{\circ} \mathrm{C}\right)$ gelagert und vor der Analy se aufgetaut und $1: 2$ mit bidest. $\mathrm{H}_{2} \mathrm{O}$ verdünnt.

\section{Prinzip}

Die Analysen wurden mittels Inductively Coupled Plasma (ICP)-Emissionsspektralanaly se ausgeführt. Das Inductively Coupled Plasma stellt für die Emissionsspektralanalyse eine relativ neue und sehr wirksame Form der Anregung dar und daher eignet sich diese Methode u.a. besonders gut zur Bestimmung von Spurenelementen in biologischen Materialien. Sehr niedrige Nachweisgrenzen (meist im Konzentrationsbereich $\mu \mathrm{g} / 1$ (ppb)) und die Möglichkeit der simultanen Bestimmung von vielen Elementen machen sie heute zu einem echten Konkurrenzverfahren der meist doch sehr aufwendigen Neutronenaktivierungsanalyse. Die sehr hohe Anregungstemperatur der Probe von etwa $8000 \mathrm{~K}$ vermeidet dabei auch die von der Atomabsorptionsspektroskopie her hinlänglich bekannten und unerwünschten Matrixeinflüsse, da bei dieser hohen Temperatur praktisch keine chemischen Verbindungen mehr vorliegen. Allerdings ist auch bei der ICP-Emissionsspektralanaly se, ähnlich wie bei der Atomabsorptionsspektroskopie, die Aerosolbildung aus der Probelösung ein sehr wichtiger Faktor (6). Für die Zerstäubung der Probe in der ICP-Emissionsspektralanalyse stehen heute zwei Verfahren zur Verfügung:

1. der pneumatische Zerstäuber und

2. der Ultraschallzerstäuber.

Im vorliegenden Fall wurde mit einem pneumatischen Zerstäuber gearbeite t. Nach der Zerstäubung wird das Aerosol in den heißen Kern eines Argonplasmas injiziert und passiert dann einen Tunnel, der von einem ringförmigen Plasma der oben erwähnten hohen Temperatur umgeben ist. Dabei werden die Probenpartikel atomisiert und angeregt, so daß sie durch ihre Strahlenemission qualitativ und quantitativ nachgewiesen werden können.

\section{Durchführung}

Die Messungen wurden an einem ICP-Emissionsspektrometer der Fa. Instruments S.A. der Type JY38 (Sequenzspektro- meter) mit angeschlossener PDP 11/03 (zur Auswertung und Prozeßsteuerung) durchgeführt. Die maximale Leistung des Hochfrequenzgenerators beträgt $1,5 \mathrm{~kW}$ bei einer Frequenz 27,12 MHz. Der Spektrometerteil besteht aus einer CzernyTurner-Anordnung mit $1 \mathrm{~m}$ Brennweite, wodurch ein spektrales Auflösungsvermögen von $0,02 \mathrm{~nm}$ erreicht wird. Als Zerstäuber wurde, wie bereits oben erwähnt, ein pneumatischer Zerstäuber Typ Meinhard eingesetzt. Durch das Vorschalten einer peristaltischen Pumpe (7) wird einerseits ein sehr viel niedrigerer Probenverbrauch gegenüber dem freisaugenden Zerstäuber von 0,9 gegen 2,5-3 ml/min erreicht und andererseits die Dichteabhängigkeit des Flüssigkeitsstromes durch die Kapillare des Zerstäubers praktisch eliminiert.

Die Messung von Al erfolgte bei $\lambda=396,152 \mathrm{~nm}(8)$. Da die von der Fa. Instruments S.A. gelieferte Software des PDP-11/03Prozeßrechners für die Auswertung und für die interne Monochromator-Shift-Kontrolle eine Referenzlinie benötigt, wurde in diesem Fall mit einer Ar-Linie von $\lambda=394,898 \mathrm{~nm}(8)$ gearbeitet.

Abbildung 1 zeigt das verwendete Auswerteverfahren und die dazu benötigten Größen.

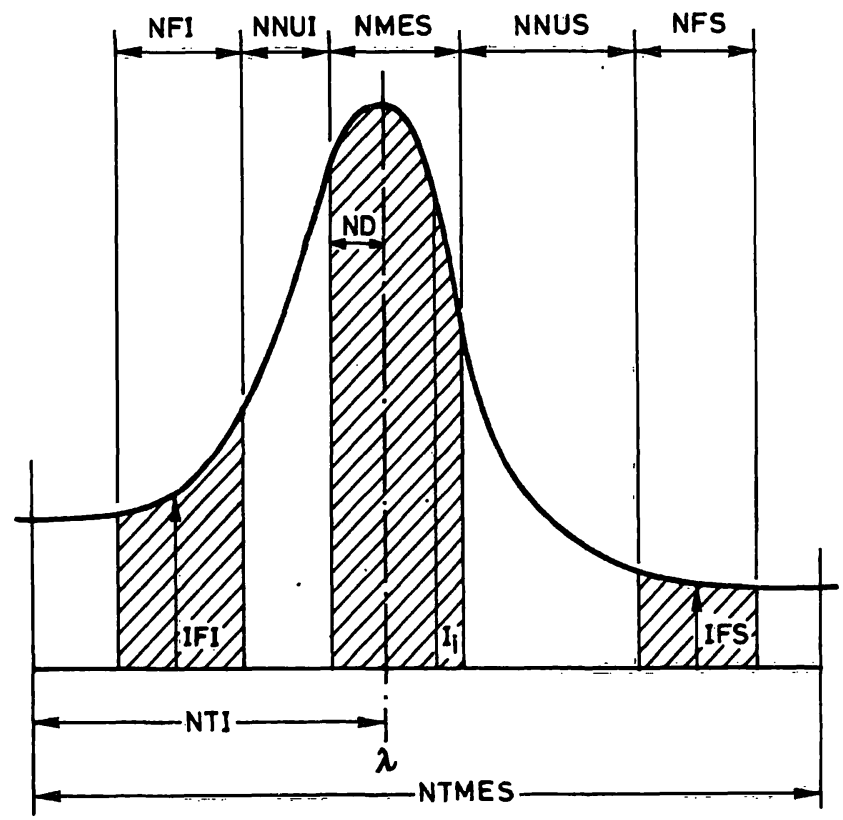

Abb. 1. Kenngrößen für die Peak-Auswertung bei der ICPEmissions-Spektroskopie.

Es bedeuten:

NTMES = Anzahl der insgesamt gemessenen Kanäle

NTI = Anzahl der gemessenen Kanäle links vom Peak

ND = Anzahl der Kanäle zur Peakflächenbestimmung links vom Peak

NMES = Anzahi aller Kanäle für die Peakflächen-

NNUI = nestimmung

= nicht zur Berechnung verwendete Kanale links (11)

NNUS = nicht zur Berechnung verwendete Kanäle rechts (8)

NFI = Anzahl der Kanäle für den linken Untergrund (8)

NFS = Anzahl der Kanäle für den rechten Untergrund (10)

In Klammern sind die für die Al-Bestimmung verwendeten Parameter angegeben. Es wurde mit einer Schrittweite von $0,005 \mathrm{~nm}$ und einer Integrationszeit von $500 \mathrm{~ms} / \mathrm{Schritt}$ gearbeitet.

\section{Ergebnisse und Diskussion}

Das Gerät wurde mit einer wäßrigen Standardlösung im Bereich von $10 \mu \mathrm{g} / 1$ (ppb) bis $10 \mathrm{mg} / 1$ (ppm) kalibriert. Es ergab sich eine lineare Standardkurve. Durch Standard- 
addition wurde überprüft, ob diese Standardkurve auch für die Bestimmung im Serum gültig ist. Es ergab sich eine.gute Ubereinstimmung in der Steigung und in den absoluten Zählraten. Darüber hinaus wurde eine analoge Standardkurve in Herrmann'scher Lösung (Riedel de Haen, Art. Nr. 38398), die in allen physikalischen und chemischen Eigenschaften dem Serum angepaßt ist, angesetzt und gemessen. Dies diente dazu, eventuell auftretende systematische Fehler durch den Einfluß von Dichte, Viskosität, Oberflächenspannung und Ionisation im Plasma überprüfen zu können. Auch hier ergab sich eine sehr gute Ubereinstimmung mit der wäßrigen Standardlösung.

Die mit diesem Verfahren erreichbare Nachweisgrenze liegt für Serum bei $10 \mu \mathrm{g} / 1$ (ppb) (definiert als $3 \mathrm{mal}$ die Standardabweichung des Untergrundes an der AlMeßlinie).

Als Mittelwert aus 20 Proben ergab sich eine Konzentration von

$$
1,6 \pm 0,6 \mu \mathrm{mol} / 1(42 \pm 16 \mu \mathrm{g} / 1(\mathrm{ppb}))
$$

mit einem Bereich der Meßwerte von 0,74-2,78 $\mu \mathrm{mol} / 1$ (20-75 $\mu \mathrm{g} / \mathrm{l}(\mathrm{ppb}))$ und einem Medianwert von 1,63 $\mu \mathrm{mol} / 1$ (44 $\mu \mathrm{g} / \mathrm{l}(\mathrm{ppb}))$.

Der in der Literatur $(3,9,10,11)$ angegebene Wert schwankt zwischen 37-1460 $\mu \mathrm{g} / 1$ (ppb). Die niedrigen Al-Konzentrationen sind dabei in besserer Ubereinstimmung mit der angenommenen begrenzten Absorption bzw. Resorption von Al durch den menschlichen Organismus. Die ICP-Emissionsspektralanalyse als empfindliche und weitgehend störungsfreie Bestimmungsmethode kann dabei einen wichtigen Beitrag zur Klärung dieser Frage liefern. Aus den Ergebnissen kann man ersehen, daß die ICP-Emissionsspektralanalyse für die
Bestimmung von $\mathrm{Al}$ in Serumproben eine hohe und ausreichende Empfindlichkeit besitzt. Die erwartete Unabhängigkeit der Bestimmungsmethode von der Matrix konnte durch die Standard-Additionsme thode überprüft werden. Diese Methode ist bei dem hier gewählten Auswerteverfahren, bei dem der jeweilige Signal-Untergrund sehr genau bestimmt und vom Gesamtsignal subtrahiert wird, erlaubt und nicht mit einem systematischen Fehler behaftet. Durch die Verwendung einer peristaltischen Pumpe, die dem Zerstäuber eine gewisse Probenmenge aufzwingt, konnte auch der Einfluß der Dichte und der Viskosität eliminiert werden. Der Probenverbrauch beträgt etwa $0,9 \mathrm{ml} / \mathrm{min}, \mathrm{d} . \mathrm{h}$. für eine Dreifachbestimmung werden etwa $3 \mathrm{ml}$ Probenlösung bzw. 1,5 ml Serum benötigt. Die Reproduzierbarkeit der Einzelmessungen ist natürlich abhängig von der Konzentration und kann bei etwa $20 \mu \mathrm{g} / 1$ (ppb) mit $\pm 8-10 \%$ angenommen werden, bei höherer Konzentration sinkt der Wert auf unter $\pm 5 \%$. Der totale Zeitbedarf für eine Analyse bestehend aus 3 Messungen je Probe beträgt etwa $5 \mathrm{~min}$, wenn alle Kalibriergrößen bereits im Programm installiert sind. Da die Gerätefunktionen sehr stabil sind, sind Zwischenkalibrierungen nicht notwendig. Durch einen weiteren Programmausbau ist zusätzlich die Möglichkeit gegeben, auch andere Elemente, z. B. Cu, $\mathrm{Fe}$ oder $\mathrm{Mn}$, aus der gleichen Probe in einem Schritt (beim Sequenzgerät allerdings nacheinander) zu messen. Insgesamt erscheint die ICP-Emissionsspektroskopie für dieses Anwendungsgebiet besser geeignet zu sein als die flammenlose Atomabsorptionsspektroskopie. Allerdings sind die Geräte sehr teuer und erfordern auch höher qualifiziertes Bedienungspersonal, so daß der Einsatz in der Routine noch problematisch erscheint.

\section{Literätür}

1. Ondreicka, R., Ginter, E. \& Kortus, J. (1966), Brit. J. Ind. Med. 23, 305-312.

2. Berlyne, G. M., Yagil, R., Ben-Ari, J., Weinberger, G., Knopf, E. \& Danovitch, G.M. (1972), Lancet $I$, 564-568.

3. Sorensen, J. R. J., Campbell, I. R., Tepper, L. B. \& Ling, R. D. (1974), Environ. Health Perspect. 7, 3-95.

4. Alfrey, A. C., Le Gendre, G. R. \& Kaehny, W. D. (1976), New Engl. J. Med. 294, 184-189.

5. Schramel, P., unveröffentlicht.

6. Schramel, P. \& Ovcar-Pavlu, J. (1979), Fresenius Z. Anal. Chem. 298, 28-31.

7. Schramel, P. (1979), Z. Lebensm. Unters. Forsch. 169 , $255-258$.

8. Winge, R. K., Peterson, V. J. \& Fassel, V. A. (1979), Appl. Spectrose. 33, 206-219.

9. Iyengar, G. V., Kollmer, W. E. \& Bowen, H. J. M. (1978), The Elemental Composition of Human Tissues and Body Fluids, Verlag Chemie, Weinheim.

10. Berlyne, G. M., Ben-Ari, J., Pest, D., Weinberger, J., Stern, M., Gilmore, G. R. \& Levine, R. (1970), Lancet II, 494-496.

11. Fuchs, C., Bransche, M., Paschen, K., Nordbeck, H. \& Quellhorst, E. (1974), Clin. Chim. Acta 52, 71-80.
Dr. Peter Schramel

Gesellschaft für Strahlen- und Umweltforschung $\mathrm{mbH}$ Institut für Angewandte Physik Physikalisch-Technische Abteilung D-8042 Neuherberg 